\title{
Modelling the migratory population dynamics of the Serengeti ecosystem
}

\author{
Janeth James Ngana ${ }^{1}$, Livingstone Serwadda Luboobi ${ }^{2}$, Dmitry Kuznetsov ${ }^{1}$ \\ ${ }^{1}$ Nelson Mandela African Institution of Science and Technology (NM-AIST), Arusha, Tanzania \\ ${ }^{2}$ Department of Mathematics, Makerere University, Kampala, Uganda \\ Email address: \\ nganaj@nm-aist.ac.tz (J. J. Ngana), luboobi@cns.mak.ac.ug (L. S. Luboobi), dmitry.kuznetsov@nm-aist.ac.tz (D. Kuznetsov)
}

\section{To cite this article:}

Janeth James Ngana, Livingstone Serwadda Luboobi, Dmitry Kuznetsov. Modelling the Migratory Population Dynamics of the Serengeti Ecosystem. Applied and Computational Mathematics. Vol. 3, No. 4, 2014, pp. 125-129. doi: 10.11648/j.acm.20140304.13

\begin{abstract}
Many ecological studies have tried to explain the animal migrations, but none has embarked on modeling the Great Migration and its impact on the migratory animals' population dynamics, in combination with food and the impact of predation. In this paper, we present a mathematical model of the four dynamic Ordinary Differential Equations of Grass, Herbivores, Lions and Crocodiles. Using secondary data covering ten years 1996-2006 we estimated the parameters in the model. The grass forage grew periodically, the herbivores population grew, the predation rate of lions grew and so did its population. But the crocodiles' population grew less. The study has shown that there was no extinction and migration continued. Herbivores population grew provided that there was enough food.
\end{abstract}

Keywords: Great Migration, Grass, Herbivores, Lions, Crocodiles, Predation, Prey, Serengeti, Masai Mara

\section{Introduction}

The Great Migration, is one of the "Seven New Wonders of the World". There is nowhere else in the world where there is such a movement of animals as immense as the wildebeests (Connochaetes taurinus), zebras (Equus burchelli) and Thomson's gazelle (Gazella thomsoni), migrating from Serengeti National Park in Tanzania to Masai Mara National Reserve in Kenya and back. Over two million animals migrate from the Serengeti to the greener pastures of the Maasai Mara. The lions, hyenas, leopards, cheetahs, crocodiles and lesser predators await the annual coming of the migration for predation.

The wildebeest (Connochaetes taurinus) migration in the Serengeti/ Mara ecosystem of Tanzania and Kenya represents an iconic example of ungulate migration and constitutes one of the most thoroughly documented animal migrations in one of the most intensively studied ecosystems on Earth (Pennycuick [7]; Maddock [4]; Fryxell et al [1]; Mduma et al. [5]; Wilmshurst et al. [10]; Wolanski and Gereta [11]; Sinclair [9]; Musiega and Kazadi [6]).

The migration is driven by a marked, highly seasonal rainfall gradient, increasing from South East to North West, coupled with strong differences in soil fertility and plant nutritional content between the grassland and savanna habitats (Maddock [4] ; Holdo et al. [3]; Holdo et al. [2]).

\section{Statement of the Problem}

Many studies have been embarking on developing the mathematical models for the rainfalls and routes patterns of wildebeests from Serengeti to Masai Mara in Kenya. Others have worked on modeling possibility of extinction of the population in case of severe drought. Not only that, but some have studied the logistic growth model in forecasting, without considering other factors like food in the model. This brings to the situation that no study has diffused itself on the issue of the modeling population dynamics by considering factors like food and predators that are crucial for the model in getting a true dimension of the migratory animals' populations at present, and for the future generation after the migration from Tanzania to Kenya and back. The case of the wildebeest, the food available for an individual can decrease because: rains don't come or too many wildebeest share too little food. The logistic model essentially assumes that only the latter is important. It should be possible to get a better model for 
the regulation of wildebeest populations by directly incorporating food supply into our model (Stratton [8]). Thus, the study aims at covering that gap and develops a mathematical model for the impact of migration.

\section{Main Objective}

To develop a population dynamics model that will reflect the Migration impact on the Serengeti Ecosystem.

\subsection{Model Formulation}

$$
\begin{gathered}
\frac{\mathrm{dG}}{\mathrm{dt}}=\mathrm{k}_{0} \cos (\theta \mathrm{t}) \mathrm{G}-\alpha \mathrm{HG} \\
\frac{\mathrm{dH}}{\mathrm{dt}=\eta \mathrm{GH}+}+\gamma \mathrm{H}-\omega \mathrm{LH}-\beta \mathrm{CH}-\psi e^{-G / H}+\sigma(1 \\
\left.-e^{-G / H}\right) \\
\frac{\mathrm{dL}}{\mathrm{dt}}=-\phi \mathrm{L}+\mathrm{vHL} \\
\frac{\mathrm{dC}}{\mathrm{dt}}=-\varepsilon \mathrm{C}+\delta \mathrm{HC} .
\end{gathered}
$$

The emigration rate, which is the migration from Tanzania to Kenya is: $\psi e^{-G / H}$ and immigration rate, that is the migration from Kenya to Tanzania is:

$$
\sigma\left(1-e^{-G / H}\right)
$$

\subsection{The variables and Parameters}

$G=$ Grass

$H=$ Herbivores

$L=$ Lions

$C=$ Crocodiles

$k_{0}=$ the rainfall constant

$\alpha=$ the efficiency rate of herbivores predation on grass

$\theta=$ the angle in radians

$\eta=$ the interaction rate of grass prey on herbivores

$\gamma=$ the intrinsic logistic rate

$\omega=$ the efficiency rate of lion predation on herbivores

$\beta=$ the efficiency rate of crocodile predation on herbivores

$\phi=$ the natural death rate of lions in the absence of prey

$v=$ the efficiency rate of lion in the presence of herbivores

$\varepsilon=$ the natural death rate of crocodile in the absence of prey

$\delta=$ the efficiency rate of the crocodile in the presence of herbivores

$\psi=$ the emigration effect rate for migratory movement from Tanzania to Kenya

$\sigma=$ the immigration effect rate for migratory movement from Kenya to Tanzania

These migration rates can be justified as follows: On the Tanzanian side, if limits are applied then as $G / H \rightarrow 0$, that's during the dry season in Tanzania ( $G$ is very small), then the animals emigrate from Tanzania to Kenya at the rate $\psi$. When $G / H \rightarrow \infty$, that's during the raining season in Tanzania( $G$ is very huge), then the animals immigrate to Tanzania from Kenya at the rate $\sigma$.

\section{The Equilibrium Points}

The following were the four equilibrium points:

1. $\left\{C=0, G=0, H=\frac{\psi}{\gamma}, L=0\right\}$

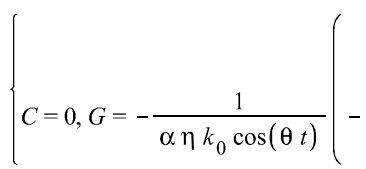

2.

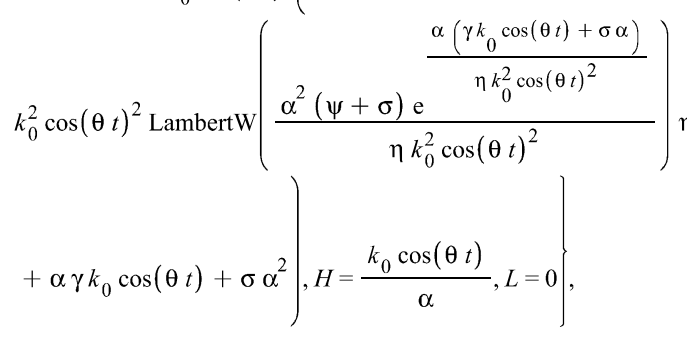

3. $\left\{C=\frac{\gamma \varepsilon-\psi \delta}{\beta \varepsilon}, G=0, H=\frac{\varepsilon}{\delta}, L=0\right\}$

4. $\left\{C=0, G=0, H=\frac{\phi}{v}, L=\frac{\gamma \phi-\psi v}{\omega \phi}\right\}$

\subsection{The Jacobian Matrices for the Model}

These were three Jacobian matrices among four corresponding to the four equilibrium points:

$J I:=\left[\begin{array}{cccc}k_{0} \cos (\theta t)-\frac{\alpha \psi}{\gamma} & 0 & 0 & 0 \\ \frac{\eta \psi}{\gamma}+\gamma+\frac{\sigma \gamma}{\psi} & \gamma & -\frac{\omega \psi}{\gamma} & -\frac{\beta \psi}{\gamma} \\ 0 & 0 & -\phi+\frac{v \psi}{\gamma} & 0 \\ 0 & 0 & 0 & -\varepsilon+\frac{\delta \psi}{\gamma}\end{array}\right]$

$J 3:=\left[\begin{array}{cccc}k_{0} \cos (\theta t)-\frac{\alpha \varepsilon}{\delta} & 0 & 0 & 0 \\ \frac{\eta \varepsilon}{\delta}+\frac{\psi \delta}{\varepsilon}+\frac{\sigma \delta}{\varepsilon} \gamma-\frac{\gamma \varepsilon-\psi \delta}{\varepsilon} & -\frac{\omega \varepsilon}{\delta} & -\frac{\beta \varepsilon}{\delta} \\ 0 & 0 & -\phi+\frac{v \varepsilon}{\delta} & 0 \\ 0 & \frac{\delta(\gamma \varepsilon-\psi \delta)}{\beta \varepsilon} & 0 & 0\end{array}\right]$ 


$$
J 4:=\left[\begin{array}{cccc}
k_{0} \cos (\theta t)-\frac{\alpha \phi}{v} & 0 & 0 & 0 \\
\frac{\eta \phi}{v}+\frac{\psi v}{\phi}+\frac{\sigma v}{\phi} & \gamma-\frac{\gamma \phi-\psi v}{\phi} & -\frac{\omega \phi}{v} & -\frac{\beta \phi}{v} \\
0 & \frac{v(\gamma \phi-\psi v)}{\omega \phi} & 0 & 0 \\
0 & 0 & 0 & -\varepsilon+\frac{\delta \phi}{v}
\end{array}\right]
$$

\subsection{The Eigenvalues of the Model}

These were the three eigenvalues among four: Eigenvalues (J1);

$$
\left[\begin{array}{c}
\gamma \\
-\frac{\gamma \phi-\psi v}{\gamma} \\
-\frac{\gamma \varepsilon-\psi \delta}{\gamma} \\
\frac{\gamma k_{0} \cos (\theta t)-\alpha \psi}{\gamma}
\end{array}\right]
$$

Conclusion: For stability of the first equilibrium point, we must have:

$$
\begin{gathered}
\gamma<0, \gamma \phi-\psi v<0, \\
\gamma \varepsilon-\psi \delta<0, \\
\gamma k_{0} \cos (\theta t)>\alpha \psi
\end{gathered}
$$

Eigenvalues(J3);

$$
\left[\begin{array}{c}
-\frac{\phi \delta-v \varepsilon}{\delta} \\
\frac{-\varepsilon \alpha+\delta k_{0} \cos (\theta t)}{\delta} \\
\frac{1}{2} \frac{\psi \delta+\sqrt{\psi^{2} \delta^{2}+4 \psi \varepsilon^{2} \delta-4 \gamma \varepsilon^{3}}}{\varepsilon} \\
-\frac{1}{2} \frac{-\psi \delta+\sqrt{\psi^{2} \delta^{2}+4 \psi \varepsilon^{2} \delta-4 \gamma \varepsilon^{3}}}{\varepsilon}
\end{array} \mid\right.
$$

Conclusion: For stability of the third equilibrium point, we must have:

$$
\begin{gathered}
\delta>0 \\
\phi \delta+v \varepsilon>0, \\
\varepsilon \alpha-\delta k_{0} \cos (\theta t)>0, \\
\varepsilon>0 \\
\psi \delta+\sqrt{\psi^{2} \delta^{2}+4 \psi \varepsilon^{2} \delta-4 \gamma \varepsilon^{3}}<
\end{gathered}
$$

$$
0, .-\psi \delta+\sqrt{\psi^{2} \delta^{2}+4 \psi \varepsilon^{2} \delta-4 \gamma \varepsilon^{3}}>0
$$

Eigenvalues(J4);

$$
\left[\begin{array}{c}
\frac{\phi \delta-v \varepsilon}{v} \\
-\frac{\phi \alpha-v k_{0} \cos (\theta t)}{v} \\
\frac{1}{2} \frac{\psi v+\sqrt{\psi^{2} v^{2}+4 \psi \phi^{2} v-4 \gamma \phi^{3}}}{\phi} \\
-\frac{1}{2} \frac{-\psi v+\sqrt{\psi^{2} v^{2}+4 \psi \phi^{2} v-4 \gamma \phi^{3}}}{\phi}
\end{array} \mid\right.
$$

Conclusion: For stability of the fourth equilibrium point, then:

$$
\begin{gathered}
v>0 \\
\phi \delta-v \varepsilon<0, \phi \alpha-v k_{o} \cos (\theta t)>0, \\
\phi>0 \\
\psi v+\sqrt{\psi^{2} v^{2}+4 \psi \phi^{2} v-4 \gamma \phi^{3}}<0, \\
-\psi v+\sqrt{\psi^{2} v^{2}+4 \psi \phi^{2} v-4 \gamma \phi^{3}}>0 .
\end{gathered}
$$

\section{The Results of Simulation Analysis of the Model with Migration}

The simplified model has thirteen parameters. The following graphs show how the data fitted the model, for Grass, Herbivores, Lions and Crocodiles against time.

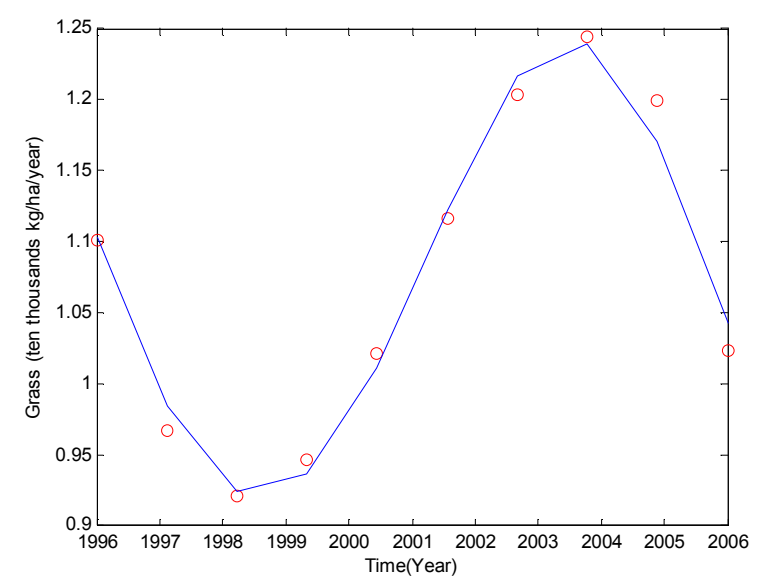

Figure 5.5(a). The graph above represents the best fitting of data for grass.

Discussion: The data is best fitting with the model. The grass grew periodically. It dropped from 1.1 in 1996 to 0.925 units of volume in 1998. Probably there was less rainfall. It then shot up to 1.25 volume units in 2003 , and started to drop again to approximately 1.04 units in 2006. 


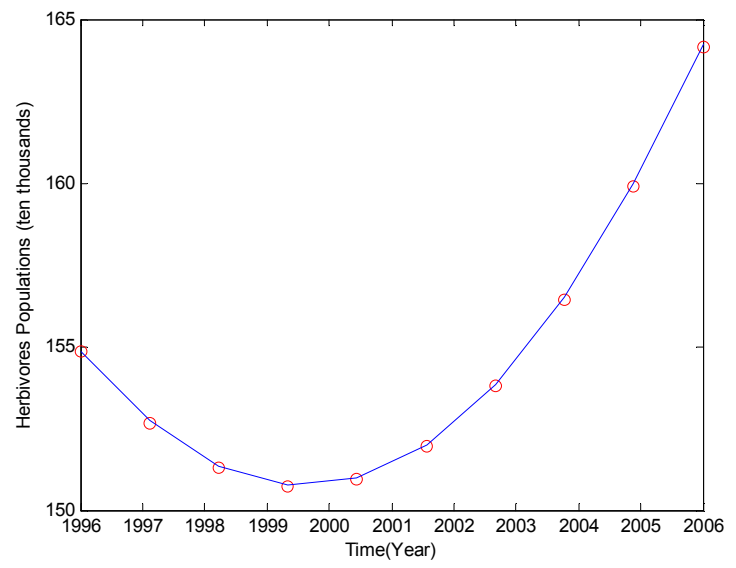

Figure 5.5(b). The graph above represents the best fitting of data for Herbivores population.

Discussion: The data is best fitting with the model. The herbivores population was $1,550,000$ in 1996 and dropped to approximately to $1,510,000$ in 1999 . Relating with the grass graph above, this was around the same period that the grass had dropped drastically in growing. But after that the population started growing up drastically again. It was around the same time that the grass volume also grew up at large.

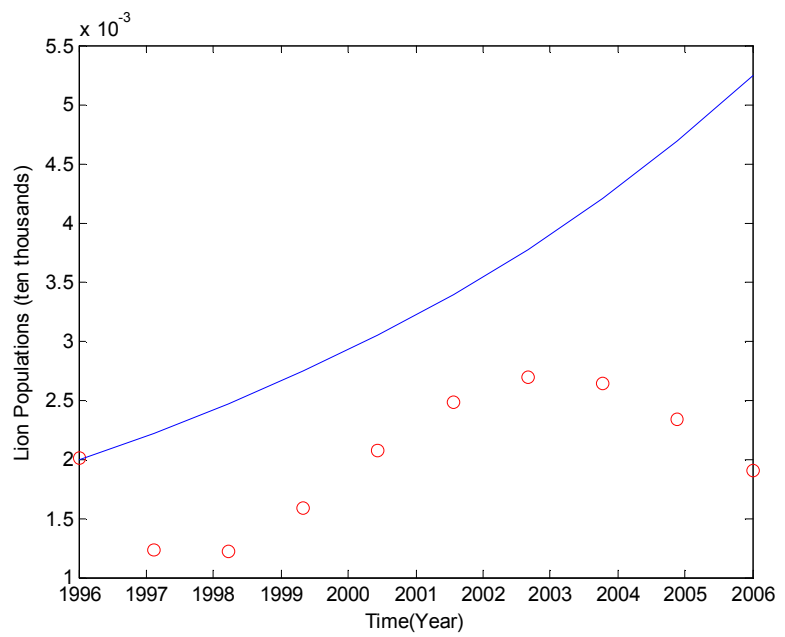

Figure 5.5(c). The graph above represents the best fitting of data for the Lions Population.

Discussion: The data does NOT well best fit with the model. This could be due to the reason that the data given was too minimal. And so the interpolated values were misleading, and hence not estimating the model properly.

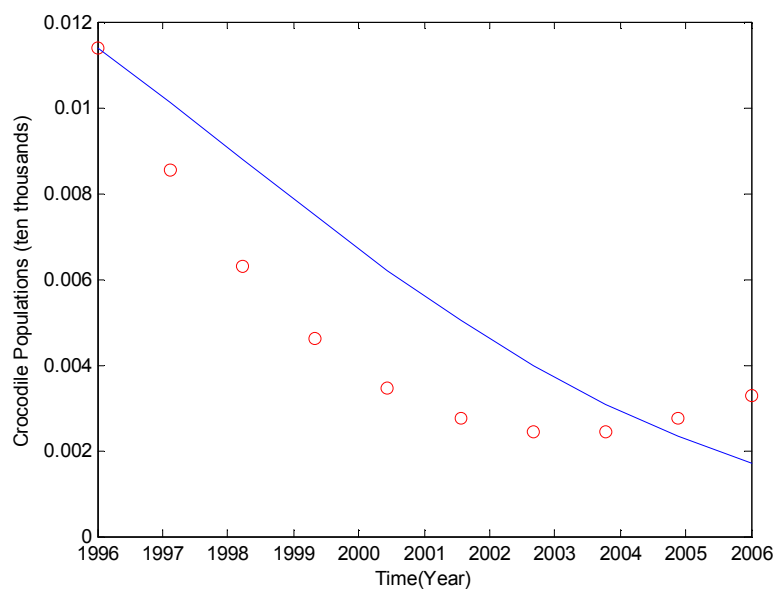

Figure 5.5(d). The graph above represents the best fitting of data for the Crocodiles.

Discussion: The data slightly best fits with the model. The graph approximately shows that the crocodile population was declining. The reason could be that maybe they don't fetch enough food. It can also be observed that it is very difficult for them to fetch the herbivores when crossing Masai-River to Kenya, and when back to Tanzania. A big reason to why their population is declining.

Conclusion: The migratory herbivores population dynamics has fortunately shown to be increasing, while the predators' population happened to decrease. There was no sign of extinction happening within the ten years, thus from 1996-2006. The population dropped around the year 2000 probably due to drought. The grass appeared to be periodically growing depending of rainfall seasoning. It is tremendously urgent for the concerned authorities to continue to control and maintain the sustainability of the existence of wildlife conservation despite some of the natural hazards which are uncontrollable. This will not only be vigilant economically for our generation, but for our future generation as well.

The following table below consist of the secondary data that was used in the simulation process for the year 1996-2006.

Table 1. Data on Grass, Herbivores, Lions and Crocodiles for the years: 1996-2006

\begin{tabular}{llllllllllll}
\hline Data & YEAR & & & & & & & & & & \\
& $\mathbf{1 9 9 6}$ & $\mathbf{1 9 9 7}$ & $\mathbf{1 9 9 8}$ & $\mathbf{1 9 9 9}$ & $\mathbf{2 0 0 0}$ & $\mathbf{2 0 0 1}$ & $\mathbf{2 0 0 2}$ & $\mathbf{2 0 0 3}$ & $\mathbf{2 0 0 4}$ & $\mathbf{2 0 0 5}$ & $\mathbf{2 0 0 6}$ \\
\hline Grass & 11027 & 9409 & 10135 & 8947 & 9147 & & 13176 & 10482 & 13405 & 11466 & 10345 \\
Herbivores & 1548554 & & & & & & & & 1544948 & & 1641630 \\
Lions & 20 & & & & & & 23 & & 19 & & \\
Crocodiles & 114 & & & & & & & 24 & & & \\
\hline
\end{tabular}

Source: Tanzania Wildlife Research Institute (TAWIRI) 


\section{References}

[1] Fryxell, J. M., Greever, J., and Sinclair, A. R. E. (1988). Causes and consequences of migration in large herbivores. Trends in Ecology and Evolution 3: 237-241.

[2] Holdo, R. M., Holt, R. D., Sinclair, A. R., Godley, B. J., \& Thirgood, S. (2011). Migration impacts on communities and ecosystems: empirical evidence and theoretical insights. Animal Migration: A Synthesis, 131-143.

[3] Holdo, R. M., Holt, R. D., and Fryxell, J.M. (2009). Opposing rainfall and pant nutritional grsdients best explain the wildebeest migration in the Serengeti. The American Naturalist, 173 (4), 431-445.

[4] Maddock, L., Sinclair, A. R. E and Norton-Griffiths, M. (1979). The Migration and Grazzing succession in Serengeti: Dynamics of an Ecosystem. 104-29. Chicago: University of Chicago Press.

[5] Mduma S.A.R, Sinclair A.R.E. AND Hiborn, R. (1999). Food regulates the Serengeti Wildbeest: a 40 -year record. Journal of Animal Ecology, 68, 1101-1122

[6] Musiega, D. E., and Kazadi, S.N. (2004). Simulating the East African Wildebeest Migration Patterns using GIS and remote sensing. African Journal of Ecology, 42 (4), 355-362.

[7] Pennycuick, C.J. (1975). On the running of gnu (Connochaetes taurinus) and other animals. Journal of Experimental Biology. 63, p.775-799.

[8] Stratton, D. (2010). Wildebeest in Serengeti: limits to exponential growth. University of Chicago Press.

[9] Sinclair, A. R. E, Arcese, P. (1995). Serengeti II: Dynamics, management and conservation of an ecosystem. University of Chicago Press, Chicago.

[10] Wilmhurst, J. F. Fryxell, J. M., Fram, B.P., Sinclair, A. R. E., Henschel, C. P. (1999). Spatial Distribution of Serengeti Wildebeest in relation to Resources. Can. J. Zool.77, 1223-1232.

[11] Wolanski, E. \& Gereta, E. (2001) .The water quality and quantity as the factors driving the Serengeti ecosystems, Tanzania. Hydrobiologia 458, 169-180. 